\title{
X-ray Microscopy and Tomography of Hydrogen Storage Materials
}

\author{
Jürgen Gluch ${ }^{1,3,4}$, Sven Niese $^{2,4}$, Lars Röntzsch $^{3}$, Ehrenfried Zschech ${ }^{2,4}$ \\ 1 Institute for Materials Science, ENano Group, TU Dresden, 01062 Dresden, Germany \\ 2 Dresden Center for Nanoanalysis (DCN), TU Dresden, 01062 Dresden, Germany \\ 3 Fraunhofer Institute for Manufacturing Technology and Advanced Materials (IFAM), Branch Lab \\ Dresden, Winterbergstr. 28, 01277 Dresden, Germany \\ 4 Fraunhofer Institute for Ceramic Technologies and Systems - Materials Diagnostics (IKTS-MD), \\ Maria-Reiche-Str. 2, 01109 Dresden, Germany
}

This paper is focused on the application of X-ray microscopy to study the change of the morphology of energy storage materials. A lab-based X-ray microscope (XCT, Carl Zeiss nano XCT 100) with a resolution better than $100 \mathrm{~nm}$ in the reconstructed volume is used for the experiments. The results are validated by experimental data obtained by scanning electron microscopy (SEM, Carl Zeiss NVision 40), transmission electron microscopy (TEM, Carl Zeiss LIBRA 200 Cs MC) and electron energy loss spectroscopy (EELS).

The redox reaction cycle of iron, $3 \mathrm{Fe}+4 \mathrm{H}_{2} \mathrm{O} \leftrightarrows \mathrm{Fe}_{3} \mathrm{O}_{4}+4 \mathrm{H}_{2}$, also known as the steam-iron-process, was historically used to produce hydrogen for industrial applications and for airship filling. Today it gains interest as a possible reaction for hydrogen storage in large quantity. The advantage is the use of cheap, non-hazardous and abundant materials. Hydrogen generation results from the reaction of highly reactive nano-sized iron powder with water steam. Hydrogen storage is performed by the reaction of magnetite powder with hydrogen. Morphological changes that do lead to coarsening of the iron powder have to be avoided in order to maintain the high reactivity and storage capacity of the material. Small powder agglomerates with a diameter of $<60 \mu \mathrm{m}$ and $<16 \mu \mathrm{m}$ were imaged with both the standard and the high-resolution optics of the X-ray microscope. A reaction chamber that fits into the path and that allows imaging of the morphological change of such iron powder agglomerates in operando was used during both oxidation and reduction under wet nitrogen and argon with 10\% hydrogen atmosphere. The reaction chamber can be heated up to $770 \mathrm{~K}$. Tilt series for tomographic reconstruction were recorded under pure nitrogen atmosphere before and after the reaction. We observed the formation of several morphologies during oxidation: At a temperature of $770 \mathrm{~K}$, an oxide shell was formed around the agglomerate. This shell was deformed by the outgassing hydrogen evolution, see Figure 1. Furthermore, the formation of oxide whiskers with a length up to $3 \mu \mathrm{m}$ was observed. The whiskers do not grow at an oxidation temperature below $620 \mathrm{~K}$. The spatial resolution of the X-ray microscope is in the range of the size of these structures, which makes it difficult to observe them and details at the particle interaction sites. Therefore, additional images were taken after the reaction using SEM and TEM, see Figure 2.

This work is funded by the European Union (ERDF) and the Free State of Saxony via the ESF project 100087859 ENano. 

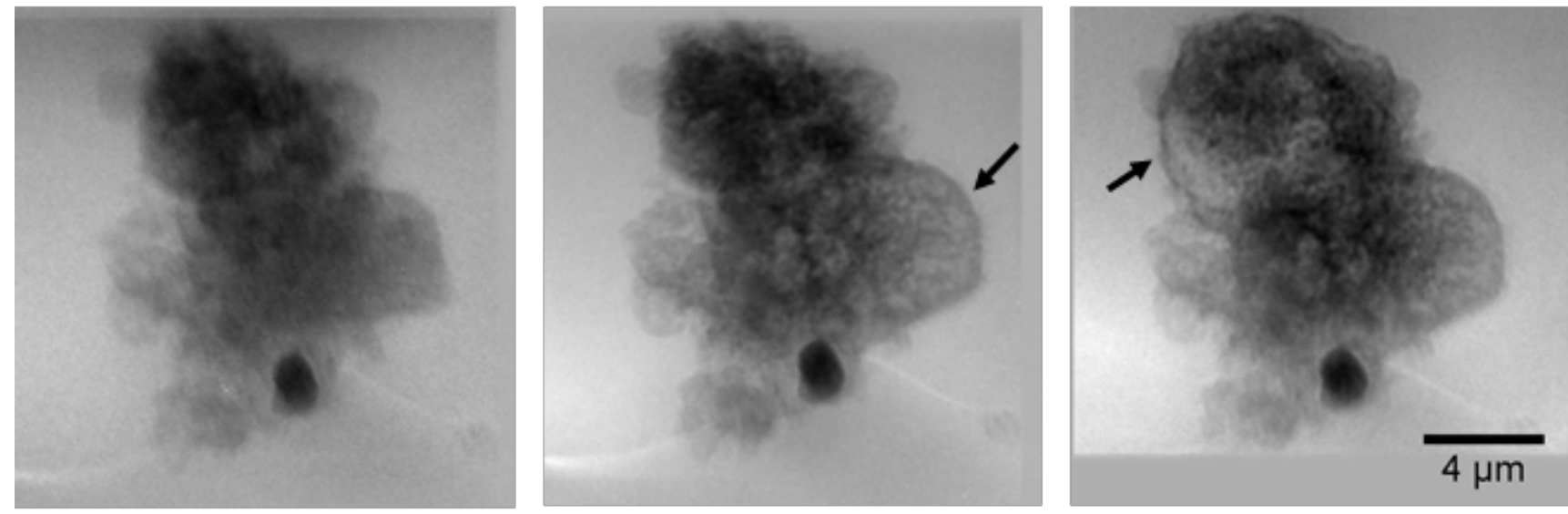

Figure 1. Sequence of $X$-ray micrographs of an iron powder agglomerate during oxidation in wet atmosphere $\left(\mathrm{N}_{2} ; 70 \% \mathrm{rh}\right)$ at a temperature of $770 \mathrm{~K}$. The observed change in morphology occurred stepwise. The images represent an averaged intensity over 25-70 frames during a phase of steady state. The arrows point to regions of oxide shell formation. Whiskers are not resolved here.

a)

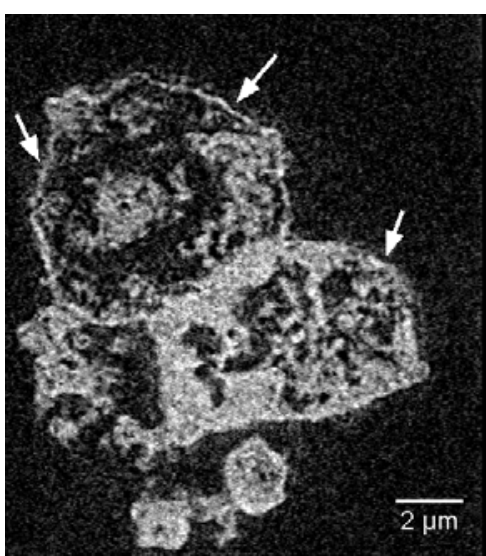

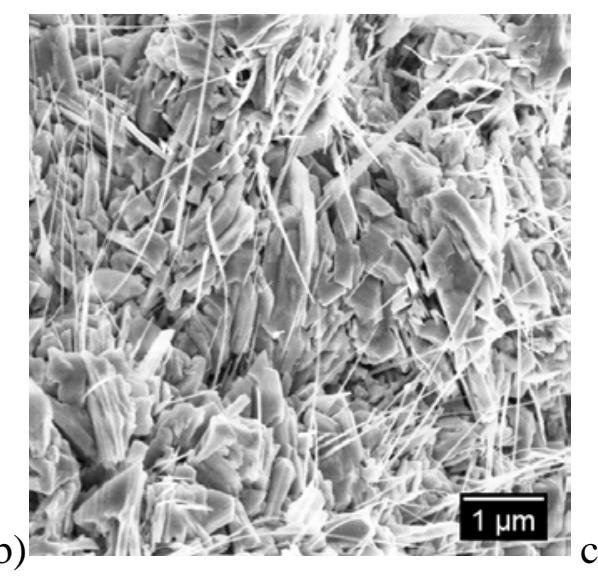

Figure 2. Oxide structures formed during the oxidation process, a) tomographic cross section through same particle as Fig. 1, the arrows point to the shell that was formed around the agglomerate particle, b) SEM view of the surface after oxidation with oxide whiskers c) TEM HAADF image of the iron oxide whiskers. 